\title{
The Impact of Baroclinic Eddy Feedback on the Persistence of Jet Variability in the Two-Layer Model
}

\author{
PABLO ZURITA-GOTOR \\ Universidad Complutense, and Instituto de Geociencia UCM-CSIC, Madrid, Spain \\ JAVIER BLANCO-FUENTES \\ Universidad Complutense, Madrid, Spain \\ EDWIN P. GERBER \\ Courant Institute, New York University, New York, New York
}

(Manuscript received 3 April 2013, in final form 31 July 2013)

\begin{abstract}
Although it is well known that the persistence of extratropical jet shifts is enhanced by a positive eddy feedback, the dynamics of this feedback is still debated. Two types of mechanisms have been proposed: barotropic mechanisms rely on changes in upper-level propagation and baroclinic mechanisms rely on the coupling between barotropic and baroclinic flow. Recent studies have suggested that barotropic models can capture key aspects of the observed jet variability but the role of baroclinic dynamics has been less explored.

This study investigates the temporal relations between barotropic and baroclinic anomalies and their eddy forcings during the internal variability of the simple two-layer quasigeostrophic model. A large correlation is found between barotropic and baroclinic anomalies and between the meridional and vertical components of the Eliassen-Palm divergence, especially at low frequency. The low-frequency variability is consistent with the baroclinic mechanism: persistent upper-level eddy momentum convergence is associated with (and precedes) persistent anomalies in the poleward eddy heat flux. In contrast, at high frequency, poleward heat flux anomalies are associated with eddy momentum divergence aloft and both eddy forcings have same-sign contributions to the upper-level eddy potential vorticity (PV) flux. In this limit the eddy PV flux is associated with wave activity transience as effective diffusivity is too small to dissipate the wave-mean flow interaction term.

The large correlation between barotropic and baroclinic anomalies implies that the low-frequency variability of barotropic flow may be affected by thermal damping when this damping is sufficiently strong. For example, zonal index persistence drops drastically in our model when baroclinicity shifts are prevented by strong thermal restoration.
\end{abstract}

\section{Introduction}

Interest in the zonal-mean variability of the atmospheric circulation dates back to the first half of the twentieth century (Rossby 1939; Namias 1950), but it revived considerably in the past two decades with the growing realization that much of the atmospheric internal variability in the extratropics can be described in terms of changes in the zonal-mean circulation. Thus, Thompson and Wallace (2000) noted that the leading

Corresponding author address: Pablo Zurita-Gotor, Departamento de Geofísica y Meteorología, Universidad Complutense, Facultad de Ciencias Físicas, Madrid 28040, Spain.

E-mail: pzurita@alum.mit.edu mode of extratropical geopotential height variability is well characterized in both hemispheres by a deep annular structure, which they called "annular modes." These modes are associated with a mass exchange between middle and high latitudes, producing a meridional seesaw in sea level pressure and a north-south displacement of the eddy-driven jet and extratropical storm tracks. Similar variability patterns are also found in a variety of atmospheric models, ranging from quasigeostrophic models (Lee and Feldstein 1996; Zhang et al. 2012) to idealized primitive equation models (Gerber and Vallis 2007; Chen and Plumb 2009) to comprehensive general circulation models (Gerber et al. 2008). It has also been noted that the atmospheric response to 
external forcing projects strongly on this mode of variability for many types of forcing (Yin 2005; Son et al. 2008b).

One can also describe the annular variability using other variables. It has become customary to define the zonal index as the principal component time series for the leading mode of variability of extratropical zonalmean zonal wind, which is essentially a proxy for the latitude of the jet (Lorenz and Hartmann 2001, hereafter LH01). The dynamical description of the annular variability is particularly simple in this framework because the vertically and zonally averaged zonal wind is forced by the eddy momentum flux alone, and damped by friction. Since the eddy momentum forcing is a very rapid process, one might expect that the decay of zonal index anomalies would be governed by the frictional time scale at times longer than the duration of synoptic life cycles. However, it was shown by LH01 that although the eddy momentum forcing is dominated by the fast, random component, it also has some power at low frequency, which is important for zonal index variability. LH01 further showed that this eddy memory is provided by the sensitivity of the eddy forcing on the mean flow, which is such that the anomalous eddy forcing tends on average to reinforce the mean flow anomalies. As a result of this positive feedback, zonal index anomalies are more persistent than indicated by the frictional time scale. The positive feedback may also explain why this dynamical pattern is a preferred mode of response of the atmospheric system. Indeed, there seems to exist a relation between the persistence of a model and the amplitude of its annular response for a given forcing (Kidston and Gerber 2010), which may be rationalized using the fluctuation-dissipation theorem (Ring and Plumb 2008).

Understanding what determines the positive feedback has thus important implications for predictability and climate sensitivity. Gerber et al. (2008) showed that the coupled general circulation models used in the Intergovernmental Panel on Climate Change (IPCC) Fourth Assessment Report (AR4) report are too persistent compared with observations in both hemispheres and seasons. Some studies have addressed the sensitivity of zonal index persistence on external parameters using idealized models (Gerber and Vallis 2007; Son et al. 2008a), but results must be interpreted with caution because changes in persistence when parameters are varied might be due to changes in the mean state rather than to changes in the feedback mechanism (Chen and Plumb 2009).

Two different types of mechanisms have been proposed to explain the observed eddy positive feedback. Barotropic mechanisms attribute the sensitivity of the eddy momentum forcing when the mean state changes to anomalous wave propagation at upper levels resulting from changes in the refraction index and/or critical layer shifts (Simmons and Hoskins 1978; Hartmann and Zuercher 1998; Chen and Zurita-Gotor 2008; Lorenz 2013, personal communication). In contrast, baroclinic mechanisms rely on changes in the wave source, as it is well known that baroclinic eddies tend to produce a westerly acceleration as they propagate away from the latitudes at which they are generated (Vallis 2006). Thus, Robinson (2000) proposed that when the eddy momentum forcing displaces the barotropic jet, the frictional drag at the surface generates baroclinicity at the location of the anomalous jet and this leads to enhanced eddy generation and eddy momentum convergence at that location, reinforcing the mean flow anomaly. More recently, Zhang et al. (2012) has argued that planetary heat transport might also be important for baroclinicity generation in the feedback loop. Observational evidence supporting the covariability of barotropic and baroclinic flow has been provided by Blanco-Fuentes and ZuritaGotor (2011, hereafter BZ11).

It is still an open question which of the two mechanisms is more important. In their observational analysis, LH01 showed that composite lagged Eliassen-Palm (EP) fluxes are consistent with the baroclinic feedback mechanism. The association between a strong upperlevel jet and cyclogenesis is well known in the synoptic literature and recent observational evidence suggests that upper-level Rossby wave breaking may foster the development of extratropical storms by strengthening the jet, potentially giving rise to persistent states and/ or storm clustering (Gómara et al. 2013). On the other hand, Barnes et al. (2010) and Barnes and Hartmann (2011) have recently shown that stirred barotropic models on the sphere can produce a positive feedback and realistic index persistence even when the wave source (the stirring) does not shift. Most convincingly, the barotropic mechanism can explain the latitudinal dependence of the eddy feedback noted by several studies: model biases in zonal index persistence appear to be related to biases in the position of the jet (Kidston and Gerber 2010; Barnes and Hartmann 2010; Arakelian and Codron 2012). Nevertheless, Simpson et al. (2013a) have recently shown that biases in the jet position may not tell the full story and other factors can also play a role for the excessive persistence of annular variability found in climate models.

The goal of this study is to test the robustness of the baroclinic feedback mechanism and to investigate the relation between barotropic and baroclinic variability more generally. This is a very challenging and largely unexplored question. Our understanding of the extratropical circulation is primarily based on two types of 
models: closure theories for the mean-state and initialvalue ("eddy life cycle") problems (Zurita-Gotor and Lindzen 2007). Yet it is not clear that the intuition gained through these models is relevant for describing the internal variability of the equilibrated time dependent problem. For instance, one defining characteristic of eddy life cycles is that eddy heat fluxes precede eddy momentum fluxes, an intuition reinforced by the climatological association between vertical convergence and meridional divergence of the Eliassen-Palm flux at upper levels (Edmon et al. 1980). However, it is not clear that this link of causality also holds for the time-dependent problem if the internal variability of baroclinicity is driven by the eddy momentum fluxes. This will be the type of question addressed in our study.

For our analysis of the extratropical internal variability, we have used a two-layer quasigeostrophic model on the beta plane, which is the simplest model that can produce a baroclinic feedback. Despite its simplicity, this model is thought to reproduce qualitatively the dynamics of the extratropical circulation (Held 2005) and has also been shown to display reasonable zonal index variability (Lee and Feldstein 1996). The simplifications of this model make it a particularly suitable tool for studying the relation between barotropic and baroclinic variability. Its minimal baroclinic decomposition eliminates any ambiguity in the definition of the baroclinic flow and its meridional symmetry removes the confounding effects of latitude - the climatological jet is always centered at midchannel and there are no differences in propagation when the jet is southward or northward of its mean position. Additionally, it is cheap to run very long simulations to enhance the low signal-to-noise ratio characteristic of this problem. This paper focuses on describing the internal variability of our model while we will study the sensitivity of zonal index persistence on external parameters in a subsequent study.

The structure of this paper is as follows. Section 2 introduces the model and formalism and shows that the internal variability of the model is in good agreement with the observational study of LH01. Section 3 analyzes the temporal relations between barotropic and baroclinic flow and between the eddy heat and momentum fluxes during the internal variability of the model at different time scales. Section 4 investigates the impact of baroclinicity damping on the variability of barotropic flow. We conclude with a summary and some discussion in section 5 .

\section{Formulation}

\section{a. Model description}

We use for this study a standard quasigeostrophic twolayer model, governed by the dynamical equations:

$$
\begin{aligned}
\frac{\partial q_{k}}{\partial t}+J\left(\psi_{k}, q_{k}\right)= & -\frac{1}{\tau_{D}}(-1)^{k} \frac{\psi_{1}-\psi_{2}-\psi_{R}}{\lambda^{2}} \\
& -\frac{1}{\tau_{F}} \delta_{k 2} \nabla^{2} \psi_{k}-\nu \nabla^{6} \psi_{k}
\end{aligned}
$$

where $q_{k}=\nabla^{2} \psi_{k}+(-1)^{k}\left(\psi_{1}-\psi_{2}\right) / \lambda^{2}+f_{0}+\beta y$ is the potential vorticity in the upper $(k=1)$ or lower $(k=2)$ layer, $\psi_{k}$ is the corresponding streamfunction, and $\lambda=$ $N H / f_{0}$ is a Rossby radius based on the layer depth $H$. This equation reflects the evolution of quasigeostrophic potential vorticity when it is advected by its balanced flow $\psi_{k}$, forced by Newtonian relaxation with time scale $\tau_{D}$ and damped by Rayleigh friction with time scale $\tau_{F}$ [in the lower layer only, as suggested by the $\delta_{k 2}$ in Eq. (1)]. Biharmonic diffusion is also used to prevent the enstrophy pileup at smaller scales. The radiative equilibrium profile is defined by a hyperbolic secant thermal wind jet, centered at midchannel $(y=0)$ with length scale $\sigma$ :

$$
-\frac{\partial \psi_{R}}{\partial y}=U_{R} \operatorname{sech}^{2}(y / \sigma) .
$$

We use the following values for our control parameters: $U_{R}=40 \mathrm{~m} \mathrm{~s}^{-1}, \sigma=2500 \mathrm{~km}, \lambda=700 \mathrm{~km}, \tau_{D}=$ 20 days, $\tau_{F}=3$ days, $\beta=1.6 \times 10^{-11} \mathrm{~m}^{-1} \mathrm{~s}^{-1}$, and $\nu=5 \times$ $10^{15} \mathrm{~m}^{4} \mathrm{~s}^{-1}$. The model is zonally periodic and bounded meridionally by rigid walls, though the domain is wide enough $\left(L_{Y}=48 \times 10^{3} \mathrm{~km}\right)$ that the dynamics is insensitive to these walls. The channel length is $L_{X}=32 \times$ $10^{3} \mathrm{~km}$. The model and parameters are the same as in Zurita-Gotor (2007) but we use a slightly higher resolution $\left(N_{X}=120, N_{Y}=240\right)$ and, most importantly, we run the model for an extended period of time to enhance the statistical power. Because the time series analyzed are so long (50000 days of semidaily data) the results presented in this paper have strong statistical significance. For instance, cross spectra are constructed using 200 independent realizations of the spectra, making a coherence squared of just 0.023 already significant at the $99 \%$ level.

Figure 1 describes the climatology of the control run. As is well known, this simple model can reproduce qualitatively the main features of the extratropical circulation. Figure 1a shows the zonal-mean zonal wind. For the parameters chosen, the upper-level wind changes little from radiative equilibrium as the baroclinicity reduction by the eddy heat flux is largely compensated by the barotropic acceleration by the eddy momentum flux. Both eddy fluxes have realistic structure, though they are stronger than observed (Fig. 1b). 

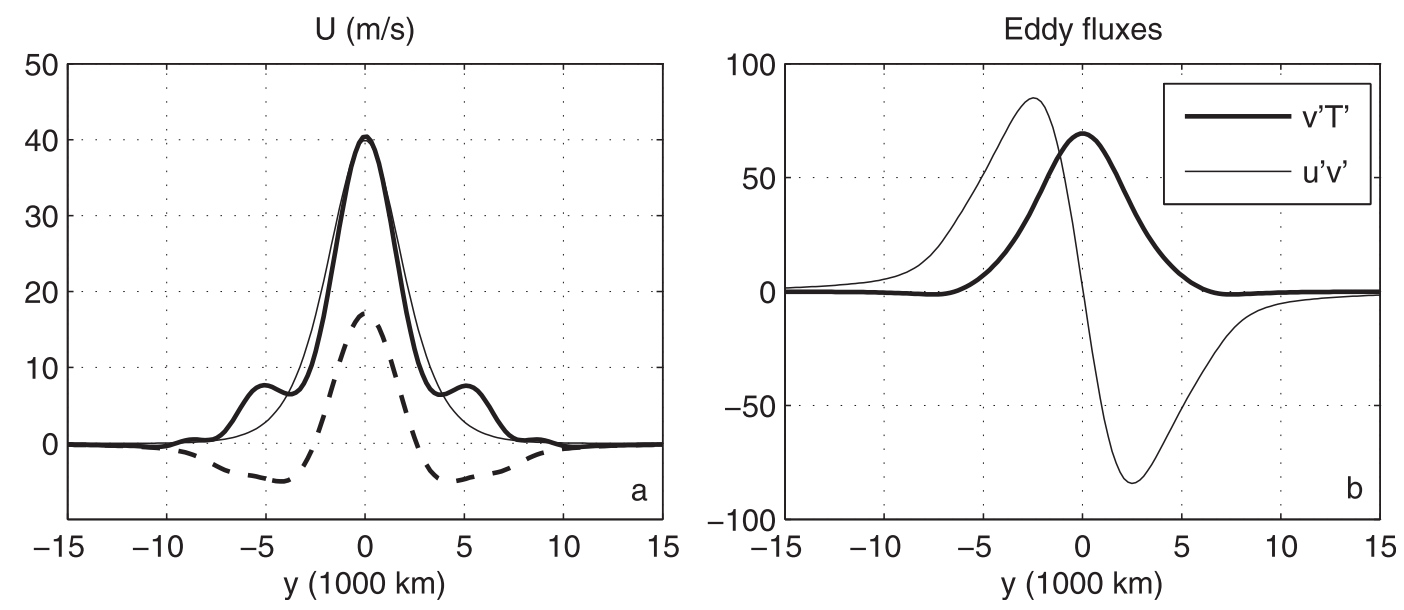

FIG. 1. Climatology of the control run: (a) zonal-mean zonal wind (thick, lower-level dashed) and radiative equilibrium jet (thin) and (b) eddy heat flux ( $\mathrm{m} \mathrm{K} \mathrm{s}^{-1}$; thick) and upper-level eddy momentum flux $\left(\mathrm{m}^{2} \mathrm{~s}^{-2}\right.$; thin).

\section{b. Internal variability}

As Lee and Feldstein (1996) showed, this model can also produce realistic internal variability of zonal-mean flow. Figure 2 describes some aspects of this variability. The leading mode of variability for the barotropic zonalmean zonal wind $\left[u_{1}\right]+\left[u_{2}\right]$ is shown with a thick line in Fig. 2a, expressed as the regression of this wind on the normalized principal component time series for its first EOF. As in observations, the leading mode represents a displacement of the barotropic jet about its mean position. However, the dynamics described by this mode is not purely barotropic as the regression of the full wind on the same index (shown with thin lines in Fig. 2a) has an equivalent barotropic structure, implying that the baroclinic wind also shifts. All this is consistent with observations.

Following LH01, we define the zonal index $z$ as the projection of the barotropic zonal-mean zonal wind on its leading EOF $\boldsymbol{v}_{U}(y)$ :

$$
z=\int\left(\left[u_{1}\right]+\left[u_{2}\right]\right) \cdot \boldsymbol{v}_{U}(y) d y .
$$

Note that since our model is perfectly symmetric about midchannel and $\boldsymbol{v}_{U}(y)$ is antisymmetric, $z$ can have no time mean: $\bar{z}=0$. We will make use of this simplification in section 4 .

The temporal evolution of the zonal index is then governed by the simple equation,

$$
\frac{\partial z}{\partial t}=m-\text { Fric }
$$

where $m=-\int \partial\left(\left[u_{1}^{*} v_{1}^{*}\right]+\left[u_{2}^{*} v_{2}^{*}\right]\right) / \partial y \cdot \boldsymbol{v}_{U} d y$ is the eddy momentum forcing and Fric $=1 / \tau_{F} \int\left[u_{2}\right] \cdot \boldsymbol{v}_{U}(y) d y$ is the frictional damping. This equation is obtained by projecting the zonal-mean equation for the barotropic zonal wind on its leading EOF $\boldsymbol{v}_{U}(y)$. Square brackets and asterisks denote instantaneous zonal-mean and eddy components.

The characteristic temporal scales of variability in our control run also compare well with observations. Figure $2 \mathrm{~b}$ shows the autocorrelation functions for the eddy momentum forcing (thin) and zonal index (thick). The former decays very rapidly and is actually negatively autocorrelated between 2 and 5 days. In contrast, the zonal index autocorrelation decays at a much slower rate, dropping to a 0.2 value after 20 days. Both are in good agreement with observed Southern Hemisphere values (cf. Figs. 4c and 4d in LH01). Gerber and Vallis (2007) show that idealized models, particularly dry ones, tend to be too persistent. Our model has a more realistic time scale in this configuration, but this is because we force it rather vigorously, leading to a stronger heat flux than observed. With weaker baroclinic forcing, our model would also be too persistent (not shown). However, note that a strong heat flux is typically required to obtain a realistic climatology in a dry model, as the circulation would otherwise tend to be too weak in the absence of latent heating during baroclinic eddy generation. Xia et al. (2011) has shown that moisture can reduce zonal index persistence in an idealized context. The results presented in this paper are qualitatively robust for other parameters, and in particular in simulations with weaker eddy fluxes and extended persistence.

We can construct an "offline model" for the zonal index by integrating Eq. (4) directly:

$$
\frac{\partial z}{\partial t}=m-\text { Fric } \approx m-\frac{z}{\tau_{Z}},
$$



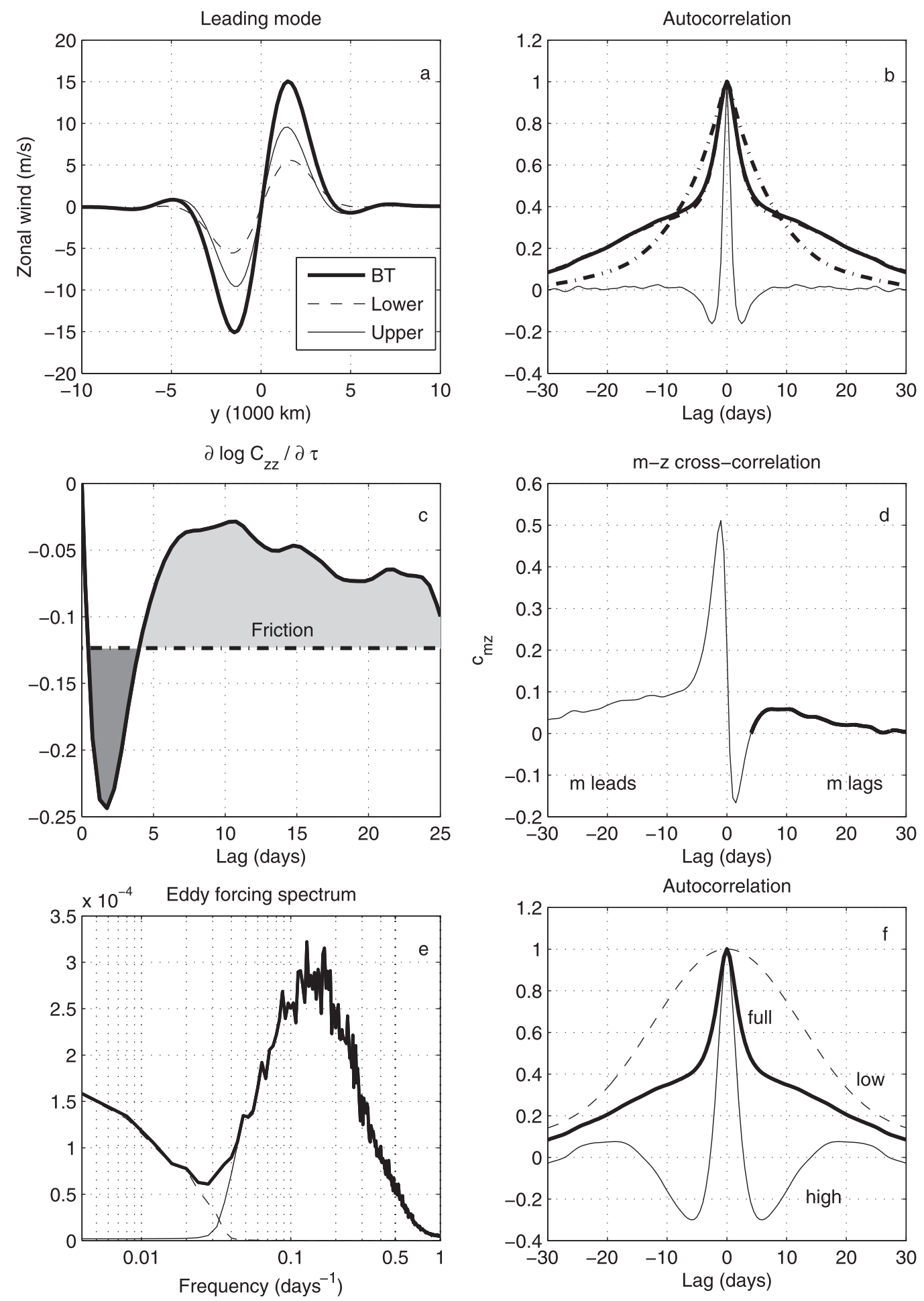

FIG. 2. (a) Leading mode of barotropic (BT) zonal-wind variability (thick solid) and regression on its normalized principal component of upper-layer (thin solid) and lower-layer (thin dashed) zonal wind. (b) Autocorrelation of eddy momentum forcing (thin solid), zonal index (thick solid), offline model approximation (thick dashed), and red noise (thick dash-dotted). (c) Logarithmic decay rate of zonal index autocorrelation, emphasizing regions of positive (light shading) and negative (dark shading) feedback. (d) Cross correlation between zonal index and eddy momentum forcing. (e) Eddy momentum forcing spectrum. (f) Autocorrelation predicted by the offline model forced by the high- and low-frequency parts of the eddy forcing, filtered as in (e). 
where the eddy forcing $m$ is taken from the simulations. This requires making the approximation (last step) that friction on the zonal index is proportional to $z$ \{in reality, friction is proportional to the lower-level wind only, and the vertical structure $\left[u_{2}\right] /\left(\left[u_{1}\right]+\left[u_{2}\right]\right)$ varies with time $\}$. We express $\tau_{Z}=2.7 \tau_{F}$ where the scaling constant is obtained from the vertical structure of the regressed wind (Fig. 2a). This is an excellent approximation, as the zonal index autocorrelation predicted by this model (thick dashed line in Fig. 2b) is nearly indistinguishable from that of the simulated $z$. This is consistent with the findings of Chen and Plumb (2009), who showed that frictional damping of the zonal index is well captured in an idealized model by projecting the annular mode/ zonal index pattern onto the lower-layer friction.

As this model illustrates, the intrinsic time scale of variability of the zonal index is much longer than friction because friction damps the lower-layer wind alone and modes are top heavy. Thus, even in the absence of any eddy memory, zonal index anomalies would decay with the long time scale $\tau_{Z} \approx 2.7 \tau_{F} \approx 8.1$ days for our control run. The autocorrelation function in this red noise limit $c_{z z}(\tau)=\exp \left(-|\tau| / \tau_{Z}\right)$ is also shown with dash-dotted line in Fig. 2b. Comparing this curve with the observed autocorrelation (solid line), we can see that the effect of eddy memory depends on lag. The eddies reduce zonal index persistence at short lags and increase it at long lags, conferring its characteristic shoulder to the observed autocorrelation. Our goal in this study is to better understand what determines the structure of eddy memory.

We can look at the effects of eddy memory in more detail modeling $m$ as a stochastic process in Eq. (5) and construct an equation for the zonal index autocovariance. This yields the following:

$$
\frac{\partial \log C_{z z}}{\partial \tau}=\frac{C_{m z}}{C_{z z}}-\frac{1}{\tau_{Z}},
$$

where $C_{z z}(\tau)=\left\langle z^{\prime}(t+\tau), z^{\prime}(t)\right\rangle$ and $C_{m z}(\tau)=\left\langle m^{\prime}(t+\tau)\right.$, $\left.z^{\prime}(t)\right\rangle$. We express correlations with a lowercase $c$ and covariances with an uppercase $C$ and define $c_{a b}$ using the convention that $a$ leads $b$ for negative values of $\tau$. Equation (6) makes it explicit that differences in the zonal index autocorrelation relative to a red noise process are due to the eddy memory (i.e., to the nonzero correlation between $m$ and $z$ when the latter leads). Figure $2 \mathrm{c}$ compares the logarithmic decay rate of the zonal index autocorrelation $\partial \log c_{z z} / \partial \tau$ with the expected decay for a damped red noise process, $1 / \tau_{Z}$. We can see that the autocorrelation decays faster than this damping rate for lags shorter than about 4 days and more slowly than this rate at longer lags, consistent with the sign change of $c_{m z}$ in Fig. 2d. The structure of the $m-z$ cross correlation is again in good agreement with observations (cf. Fig. 5 in LH01), displaying in particular a region with positive values at long positive lags. As LH01 note, it is this positive $c_{m z}$ correlation that accounts for the slowerthan-frictional zonal index decay at long lags, which LH01 attribute to a positive eddy feedback. Simpson et al. (2013b) have recently proposed estimating the strength of this feedback as the long-lag value of the $C_{m z} / C_{z z}$ ratio, consistent with Eq. (6). On the other hand, the negative values of $c_{m z}$ at short lags are also in good agreement with observations, though the correlations simulated by our model are significantly larger and the transition from negative to positive $c_{m z}$ more abrupt than observed, which gives rise to a noticeable shoulder in the simulated zonal index autocorrelation. This shoulder is much less evident in other parameter regimes, but the transition from a negative to a positive $c_{m z}$ is robust for all simulations we have analyzed.

Finally, the structure of the eddy forcing power spectrum $M^{*} M$ is shown in Fig. 2e (we use uppercase letters to denote Fourier transforms of the corresponding lowercase variables and asterisks for their complex conjugate forms). Because our simulations are very long, this spectrum is fairly well resolved, even at low frequency. The spectrum consists of a broad peak centered at frequencies between $1 / 5$ and $1 / 10$ days $^{-1}$, associated with the high-frequency eddies, and a secondary low-frequency peak conferring some redness to the power spectrum. This is again in qualitative agreement with observations (cf. Fig. 4b in LH01), though the spectral gap separating the two peaks is not as clean in the observed data. However, this might be partly due to the shorter record length/ lower spectral resolution in observations.

By virtue of Wiener's theorem, the eddy momentum forcing autocovariance and the eddy forcing power spectrum form a Fourier pair, so the $C_{m m}$ structure may be understood as the sum of the Fourier transforms of the two spectral peaks noted earlier. For the purpose of discussion, it is illustrative to model these two peaks as a sharp (note the logarithmic scale) Gaussian with width $\delta_{\omega 0}$ centered at zero frequency, and a broader Gaussian with width $\delta_{\omega e}$ centered at the characteristic eddy frequency $\omega_{e}$. The Fourier transform of the first Gaussian would be another Gaussian, slowly decaying as $\exp \left(-\delta_{\omega 0}^{2} \tau^{2}\right)$. As noted by LH01, it is this low-frequency peak that accounts for the extended memory of zonal index at long lags. However, because this peak only captures a small fraction of the total energy in the eddy forcing spectrum, the full eddy forcing autocovariance $C_{m m}$ is dominated by the contribution associated with the second peak at $\omega_{e}$, which varies as $\exp \left(-\delta_{\omega e}^{2} \tau^{2}\right) \cos \left(\omega_{e} \tau\right)$. Although this is the dominant contribution to $C_{m m}$, for $\delta_{\omega e} \gg$ $\delta_{\omega 0}$ this term will decay faster than the low-frequency 
contribution, which will eventually dominate at long lags. Figure $2 \mathrm{f}$ shows that this mathematical description is broadly consistent with the structure of zonal index autocorrelation produced by the offline model when this model is forced by the low- and high-frequency components of the full $\mathrm{m}$ forcing, filtered as in Fig. 2e.

This mathematical analysis shows that the eddy forcing autocorrelation will exhibit oscillatory behavior whenever $\delta_{\omega e}<\omega_{e}$ (otherwise the autocorrelation would decay faster than a period). Although the Gaussian representation is not a good model for the high-frequency eddy forcing spectrum, this is a robust result and it can be shown more generally using spectral windows that the oscillatory behavior in $c_{m m}$ is associated with the spectral suppression of $m$ at low frequencies. We provide a physical interpretation for this result in section $3 \mathrm{c}$.

In conclusion, the good agreement between the diagnostics presented in this section and the observational analysis of LH01 gives us some confidence that the dynamics of zonal index variability in our model might also be relevant for the real atmosphere. In the next section, we analyze in more detail the dynamics of internal variability in our model.

\section{Dynamics of internal variability}

The baroclinic feedback mechanism proposed by Robinson (2000) consists of two steps. Following the formation of an anomalous barotropic jet by the anomalous eddy momentum fluxes, zonal-mean baroclinicity is generated as friction damps the lower-layer wind. This then leads to enhanced eddy generation at the location of the baroclinic anomaly and to enhanced eddy momentum convergence as the eddies propagate meridionally away from that location, which reinforces the original barotropic anomaly. We have recently shown (BZ11) that the observed variability of zonal-mean baroclinicity in the Southern Hemisphere is consistent with Robinson's mechanism. In this section we extend the observational analysis of BZ11 to our model and further investigate the temporal relations between the eddy fluxes.

\section{a. Baroclinicity variability}

Figure 3 a shows the leading two modes of variability for the zonal-mean baroclinicity $-\partial[\theta] / \partial y$, which may be described as a baroclinicity shift (thick solid, 35.2\% variance) and a sharpening of the climatological baroclinicity (thin dashed, 22.4\% variance), respectively. Repeating the EOF analysis with filtered data shows that the ordering of these two modes is robust for all time scales but the shift is most dominant at low frequency, while at high frequency the two modes are poorly separated (not shown). We can construct an equation for the baroclinicity shift by projecting the ( $y$ differentiated) thermodynamic equation onto the leading baroclinicity $\operatorname{EOF} \boldsymbol{v}_{\theta}(y)$ :

$$
\frac{\partial b}{\partial t}=F\left[v^{*} \theta^{*}\right]+F[\mathrm{MMC}]-\frac{b}{\tau_{D}}=h-\frac{b}{\tau_{D}},
$$

where $b=\int-\partial[\theta] / \partial y \cdot \boldsymbol{v}_{\theta} d y$ is the PC of the leading baroclinicity mode (for brevity, hereafter this will be termed "the baroclinicity"), $F\left[v^{*} \theta^{*}\right]=\int \partial^{2}\left[v^{*} \theta^{*}\right] / \partial y^{2} \cdot \boldsymbol{v}_{\theta} d y$ is the forcing by the eddy heat flux divergence and $F[\mathrm{MMC}]=\int \partial[w] / \partial y \Theta_{z} \cdot \boldsymbol{v}_{\theta} d y$ is the forcing by the mean meridional circulation (MMC). The net dynamical warming is given by $h=F\left[v^{*} \theta^{*}\right]+F[\mathrm{MMC}]=$ $\int \partial\left[w^{\text {res }}\right] / \partial y \Theta_{z} \cdot \boldsymbol{v}_{\theta} d y$, where $\left[w^{\text {res }}\right]$ is the residual vertical velocity, and diabatic damping is given by $Q=-b / \tau_{D}$. Since this equation is formally identical to Eq. (5) for the zonal index, we can likewise write the following:

$$
\frac{\partial \log C_{b b}}{\partial \tau}=\frac{C_{h b}}{C_{b b}}-\frac{1}{\tau_{D}} .
$$

Figure $3 \mathrm{~b}$ shows that the autocorrelation function of the dynamical baroclinicity forcing $h$ has a similar structure as $m$, with sharp decay and large negative autocorrelations between 1 and 4 days. While $b$ decays more slowly than $h$, it still decays much faster than the red noise process $c_{b b}(\tau)=\exp \left(-|\tau| / \tau_{D}\right)$, obtained in the limit in which $h$ has no memory. In fact, Fig. $3 b$ shows that $b$ decays faster than $z$ despite its longer damping scale. This is confirmed by Fig. 3c, which shows that (i) $b$ is subject to stronger negative dynamical forcing than $z$ at short lags (cf. Fig. 2c) and (ii) no region with positive dynamical forcing is apparent in this case. Consistent with the absence of a positive dynamical feedback for $b$, there are important qualitative differences with the analysis of barotropic variability. First, there is not a positive bump in the $C_{h b}$ correlation at positive lags (thick line in Fig. 4a). Additionally, the $h$ spectrum exhibits no redness at low frequency (thick line in Fig. 4b), as there is a cancellation between the redness of its eddy heat flux and MMC components. In the absence of a positive feedback, the long-lag decay rate of $b$ is close to the diabatic time scale.

We next study the relation between the variability of $b$ and $z$. Figure $3 \mathrm{~d}$ shows that both time series are very strongly correlated, with the maximum correlation obtained for short, $O(1$ day) leads of the barotropic anomalies. This lag is much smaller than the decorrelation time scales of either $b$ or $z$, suggesting that it is not possible to disentangle the variability of $b$ and $z$ and that they should be regarded as essentially the same 

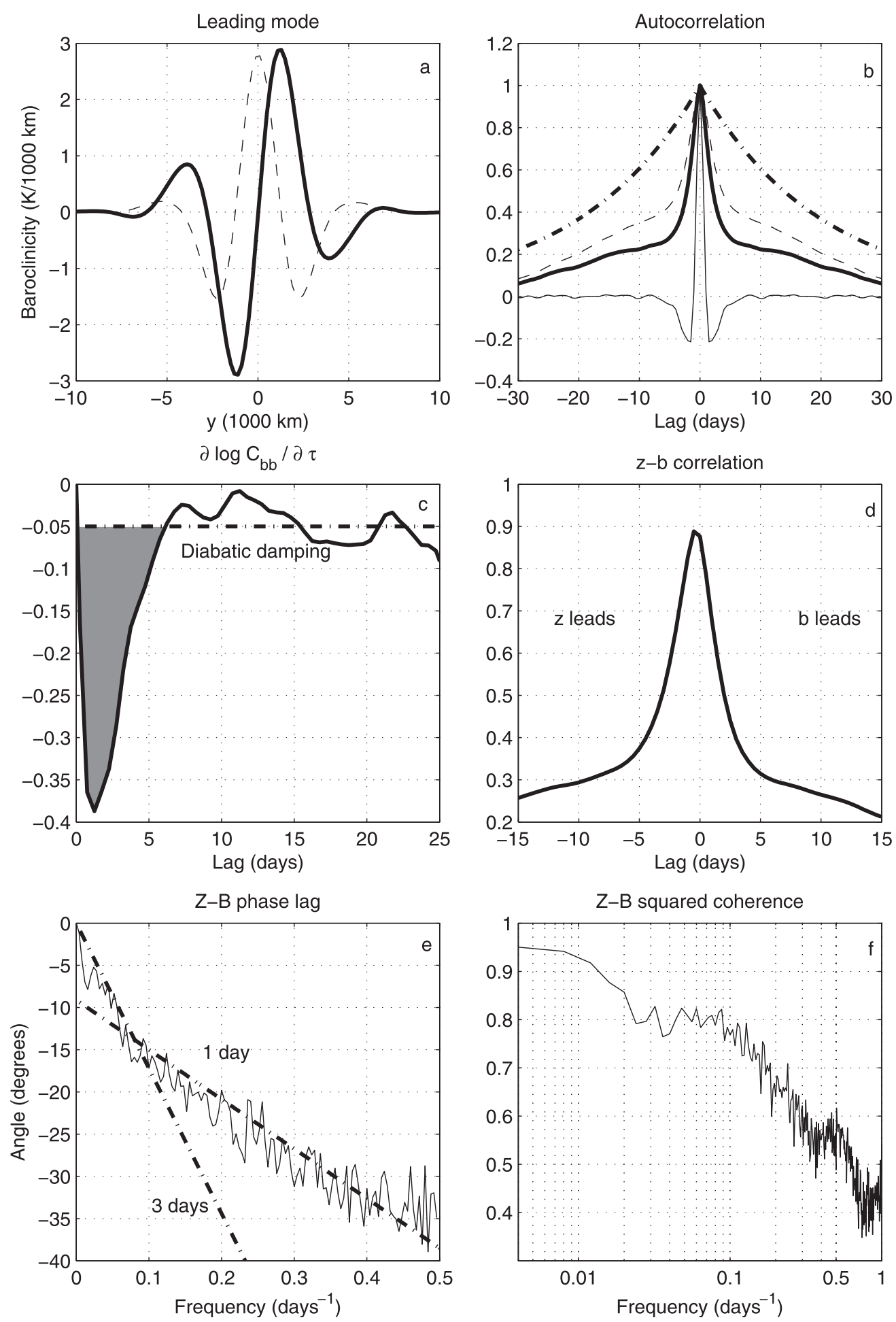

FIG. 3. (a) Leading two modes of baroclinicity variability (amplitude corresponds to one standard deviation of the corresponding principal component). (b) Autocorrelation of leading baroclinicity mode (thick solid), red noise (thick dash-dotted), zonal index (dashed), and dynamical forcing (thin solid). (c) Logarithmic decay rate of baroclinicity autocorrelation. (d) Lagged correlation between the leading baroclinicity mode and zonal index. (e) Phase difference in the $Z-B$ cross spectrum and slopes corresponding to 1- and 3-day lags. (f) Squared coherence of $Z-B$ cross spectrum. 

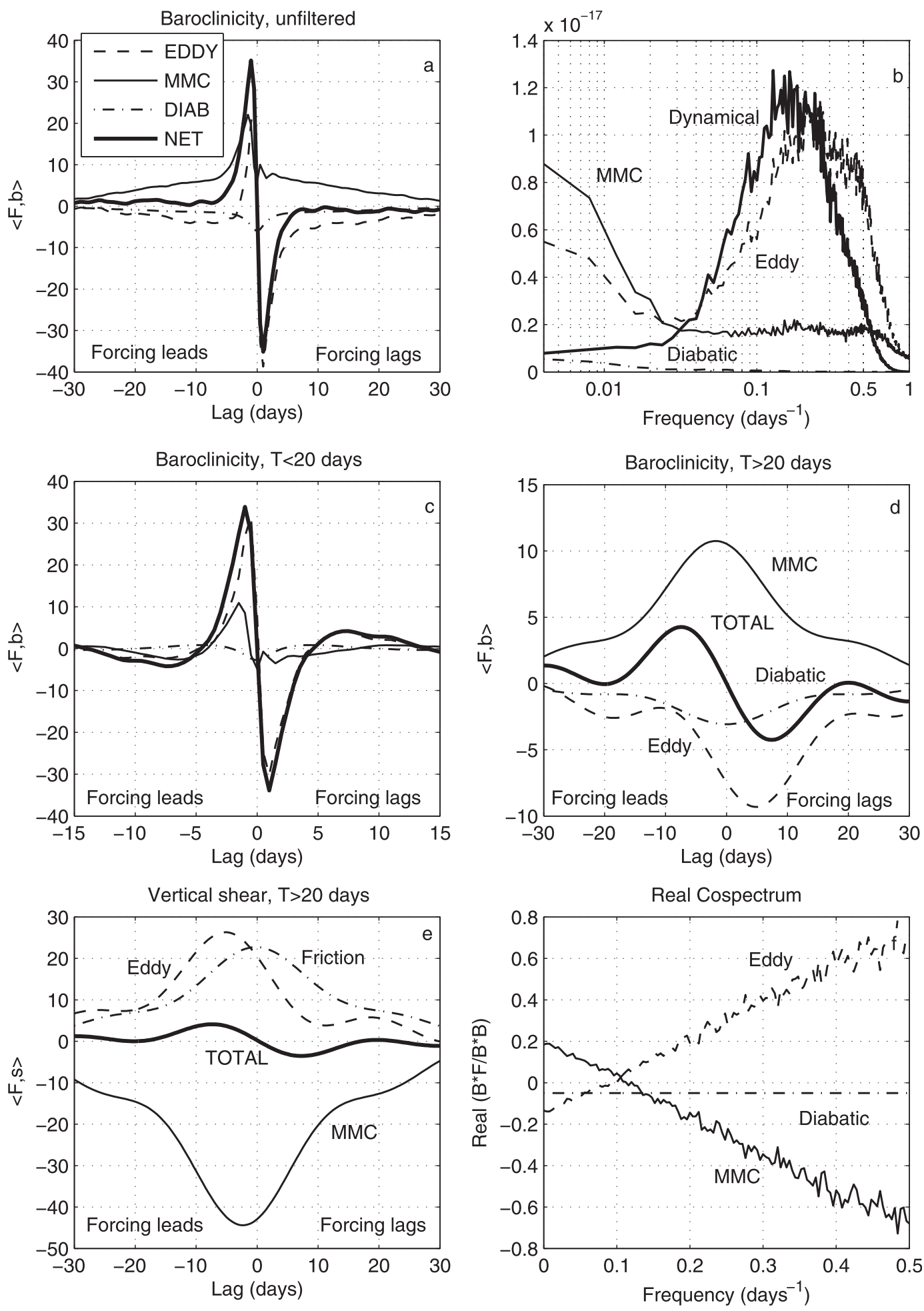

FIG. 4. (a) Lagged covariance between baroclinicity and its forcings: eddy heat flux (dashed), mean meridional circulation (solid), diabatic heating (dash-dotted) and their sum (thick solid), with units of K (1000 km) ${ }^{-2} \mathrm{day}^{-1}$. (b) Power spectra of the baroclinicity forcing terms. (c) As in (a), but using high-pass-filtered data. (d) As in (a), but using low-pass-filtered data. (e) As in (a), but for the vertical shear balance (eddy momentum flux dashed, mean meridional circulation solid, friction dash-dotted). (f) Real part of the normalized $B-F$ cross spectrum $\left(\right.$ day $\left.^{-1}\right)$. 
phenomenon. Although the lag of maximum $b-z$ correlation is small and close to the sampling rate, it is also unambiguously negative: the sign of this lag remains robust when subdivisions of the full time series are considered as well as in other simulations analyzed (not shown). We have also computed the complex cross spectrum between $B$ and $Z$. The phase lag between $B$ and $Z$ is consistent with a $b$ delay of about 1 day over a broad frequency range, but somewhat longer at low frequency (Fig. 3e). The coherence is very high and largest at the low frequency (Fig. 3f).

Following BZ11, we compute the characteristic life cycles of the $b$ anomalies regressing each of the forcing terms in Eq. (7) against $b$. Figure 4a shows that the eddy heat flux forcing and MMC forcing are both important but the diabatic term is much smaller. Figure $4 \mathrm{~b}$ shows the power spectra of the different forcing terms: we can see that while the eddy heat flux is clearly dominant at high frequency, the MMC forcing becomes larger at low frequency. This is consistent with the observational results of BZ11, who showed that the dynamics of the baroclinicity anomalies is sensitive to frequency. The same is true in our model. Figures $4 \mathrm{c}$ and $4 \mathrm{~d}$ show the life cycles of high ( $T<20$ days)- and low ( $T>20$ days)frequency baroclinic anomalies, obtained filtering all terms in Eq. (7) using a 121-weight Lanczos filter. At high frequency (Fig. 4c) the baroclinicity essentially responds to the eddy heat flux forcing. In contrast, lowfrequency baroclinicity anomalies are forced by the MMC warming and damped by the eddy heat flux and, to a lesser extent, the diabatic heating. To understand the source of this MMC forcing, we can compute an evolution equation for the scaled zonal-mean vertical shear $\alpha\left(\left[U_{1}\right]-\left[U_{2}\right]\right)$, where $\alpha=f_{0} \Theta_{0} /(g H)$ is the constant factor relating baroclinicity and vertical shear in the thermal wind relation for this model, and $\Theta_{0}$ is a reference temperature and $g$ the gravitational acceleration. From the momentum equation:

$$
\frac{\partial s}{\partial t} \approx F\left[u^{*} v^{*}\right]+F\left[\mathrm{MMC}_{U}\right]+\frac{s}{\tau_{F}},
$$

where $s=\int \alpha\left(\left[U_{1}\right]-\left[U_{2}\right]\right) \cdot \boldsymbol{v}_{\theta} d y$ exactly equals $b$ by the thermal wind constraint, $F\left[u^{*} v^{*}\right]=-\int \alpha \partial\left[u_{1}^{*} v_{1}^{*}-\right.$ $\left.u_{2}^{*} v_{2}^{*}\right] / \partial y \cdot \boldsymbol{v}_{\theta} d y$ is the eddy momentum forcing, $F\left[\mathrm{MMC}_{U}\right]=\int 2 \alpha f_{0}\left[v_{a}\right] \cdot \boldsymbol{v}_{\theta} d y$ is the MMC forcing, and $\left[v_{a}\right]$ is the upper-layer ageostrophic meridional velocity, which was diagnosed from the model output. Note that Eq. (9) is only approximate because we neglected horizontal hyperdiffusion.

The characteristic life cycle of low-frequency vertical shear anomalies is shown in Fig. 4e. These anomalies are forced by the eddy momentum flux and friction, while they are damped by the MMC forcing. Putting Figs. 4d and $4 \mathrm{e}$ together, the life cycles of the baroclinicity and shear anomalies may be described as follows: (i) the eddy momentum flux, stronger in the upper layer, forces a vertical shear anomaly; (ii) this anomaly is enhanced/ maintained by the frictional damping of the lower-layer wind; (iii) an anomalous mean meridional circulation (Ferrel cell shift) is driven that damps the shear anomaly and forces a baroclinicity anomaly to maintain thermal wind balance; and (iv) the baroclinicity anomaly is damped by the combined effects of the downgradient eddy heat flux and diabatic heating.

Our analysis is in good agreement with that of BZ11 using Southern Hemisphere data, except that planetary waves are also present in that case [producing an additional forcing of baroclinicity, largely balanced by the synoptic eddy heat flux; see also Thompson and Birner (2012)] and diabatic heating is quantitatively more important. However, both in observations and in our model the dynamics of low-frequency baroclinicity anomalies is consistent with the feedback mechanism proposed by Robinson (2000). We can estimate more precisely the frequency range over which this dynamics is at work in our model by computing the cross spectrum between baroclinicity and its various forcing terms. Figure $4 \mathrm{f}$ shows the real part (in-phase component) of this cross spectrum. At high frequency, baroclinicity anomalies are forced by the eddy heat flux and damped by the MMC forcing. [The imaginary, out-of-phase component of the cross spectrum, associated with propagation, is larger and dominated by the eddy heat flux (not shown)]. The reverse is found at low frequency, when baroclinicity anomalies are forced by the MMC and damped by the "diffusive" eddy heat flux. The transition is found at frequencies of about $1 / 10$ days $^{-1}$ in our model.

\section{b. Internal variability of the eddy fluxes}

The second step of Robinson's argument involves enhanced eddy generation and enhanced eddy momentum convergence at the location of the anomalous baroclinicity. We already showed above that the eddy heat flux behaves diffusively and tends to damp the baroclinic anomalies (implying enhanced eddy generation) when these anomalies have periods longer than about 10 days. For the mean circulation, the relation between eddy generation and eddy momentum convergence is well established (Vallis 2006, chapter 12) as waves generated baroclinically propagate vertically into the upper troposphere and then meridionally away from the generation region, converging momentum at upper levels. Thus, the vertical EP convergence at upper levels is partially compensated by its meridional divergence, 
and the net upper-level eddy PV flux is reduced. Figure $1 \mathrm{~b}$ illustrates this type of behavior in our model.

To investigate the relevance of these ideas for zonal index persistence in our model, we regress in Figs. 5a and 5b the meridional and vertical components of the upperlevel EP divergence,

$$
\begin{aligned}
\partial_{y} F_{y} & =-\frac{\partial}{\partial y}\left[u_{1}^{*} v_{1}^{*}\right], \quad \partial_{z} F_{z}=-\frac{f_{0}}{\Theta_{z} H}\left[v_{1}^{*} \theta^{*}\right], \\
{\left[v_{1}^{*} q_{1}^{*}\right] } & =\nabla \cdot F=\partial_{y} F_{y}+\partial_{z} F_{z},
\end{aligned}
$$

on the normalized $z$ time series. Both regressions exhibit a clear dipolar pattern but the picture is somewhat complicated: while at long positive and negative lags $\partial_{y} F_{y}$ and $\partial_{z} F_{z}$ tend to compensate each other, the contrary is true at short lags. Since this suggests that the relation between the eddy fluxes might be different at different time scales, we also show in Fig. 5 the regression of the eddy fluxes on the slow and fast components of the $z$ time series (filtered using a 40-day cutoff Lanczos filter). For the slow variability (Figs. 5c,d), the climatological relation holds and there is an association between vertical convergence and meridional divergence of the Eliassen-Palm fluxes. In contrast, for the fast variability (Figs. 5e,f), there is a positive correlation between the meridional and vertical EP divergence, so that both components produce contributions of the same sign to the eddy potential vorticity flux. As a result, the $\left[v_{1}^{*} q_{1}^{*}\right]$ variability is dominated by the shorttime response (Fig. $5 \mathrm{~g}$ ), much more so than that of its barotropic and baroclinic components independently. We discuss the origin of this impulsive eddy PV flux in section $3 \mathrm{c}$.

The lagged cross correlation between $\partial_{y} F_{y}$ and $\partial_{z} F_{z}$ (with both projected on the direction of $\boldsymbol{v}_{U}$ ) is consistent with these results (Fig. 5h). The correlation is dominated by the fast variability, displaying a sharp, positive peak for short leads of $\partial_{y} F_{y}$, surrounded by a broader shoulder with negative cross correlation. It is noteworthy that the region with negative correlation is stronger and more persistent on the right of the peak (see also Fig. $7 \mathrm{a}$ ), when $\partial_{y} F_{y}$ leads. This is consistent with the phase relation between the $\partial_{y} F_{y}$ and $\partial_{z} F_{z}$ anomalies in Figs. 5c and $5 \mathrm{~d}$, the former leading the slow variability of $z$ and the latter lagging. We can thus conclude from Fig. 5 that the $\partial_{y} F_{y}$ anomalies drive the variability of wavemean flow interaction both at high frequency, when they precede $\partial_{z} F_{z}$ anomalies of the same sign, and at low frequency, when they precede anomalies in $\partial_{z} F_{z}$ of the opposite sign. To illustrate this, Figs. $6 a$ and $6 b$, show sample time series of both $\nabla \cdot F$ components for low- and high-pass-filtered data ( $T>40$ days and $T<7$ days), respectively. The correlation between the two components of the EP divergence can be appreciated visually and is particularly striking for the low-frequency data.

The low-frequency phase relation, with anomalous eddy momentum flux convergence preceding positive anomalies in the eddy meridional heat flux, is consistent with the baroclinic feedback mechanism. To assess the robustness of this relation, we have computed the complex cross spectrum between $\partial_{y} F_{y}$ and $\partial_{z} F_{z}$. The magnitude of the cross spectrum mirrors the spectra of the eddy forcing presented in previous sections, with a synoptic peak and a second peak at low frequency (Fig. 6d, thin line). However, the phase lag is very different in both regions of the spectrum, with the $\partial_{y} F_{y}$ anomalies leading $\partial_{z} F_{z}$ anomalies of the same (opposite) sign over the former (latter) as discussed above (Fig. 6c). The squared coherence is very significant and highest at the low frequency (Fig. 6d, thick line), consistent with the large correlations noted in Fig. 6a.

\section{c. The short time response}

As shown above, at low-frequency the meridional and vertical components of the Eliassen-Palm divergence tend to cancel each other, consistent with well established theories of the extratropical circulation (Vallis 2006). In contrast, for the fast variability the anomalous eddy fluxes tend to have contributions of the same sign to the upper-level eddy PV flux, which seems harder to understand. The short time relation between the eddy fluxes is very robust: the lagged correlation between $\partial_{y} F_{y}$ and $\partial_{z} F_{z}$ at midchannel features a very similar structure to that described for their projection on the leading EOF above (cf. the thin and thick lines in Fig. 5h). In contrast, note that the extended anticorrelation for positive $\partial_{y} F_{y}$ leads that we associate with a positive feedback is only really apparent for the EOF projection (this is best appreciated using a logarithmic scale, see Fig. 7a). To investigate the short time response, we will focus on the variability of the EP fluxes at midchannel (where climatological values are largest) for the remainder of this section, but note that the results are very similar in EOF space.

The instantaneous values of the wave-mean flow interaction terms are typically much larger than their timemean values, as illustrated by the histograms of semidaily values of the barotropic, baroclinic, and total upper-level eddy PV flux at midchannel in Fig. 7b. The histograms reveal that the positive sign of the climatological meridional EP divergence, opposite to that of $\partial_{z} F_{z}$, results from averaging a highly variable field, for which positive and negative values are in fact almost equally likely. It is also interesting that the instantaneous barotropic and baroclinic contributions to the eddy PV flux are quite 

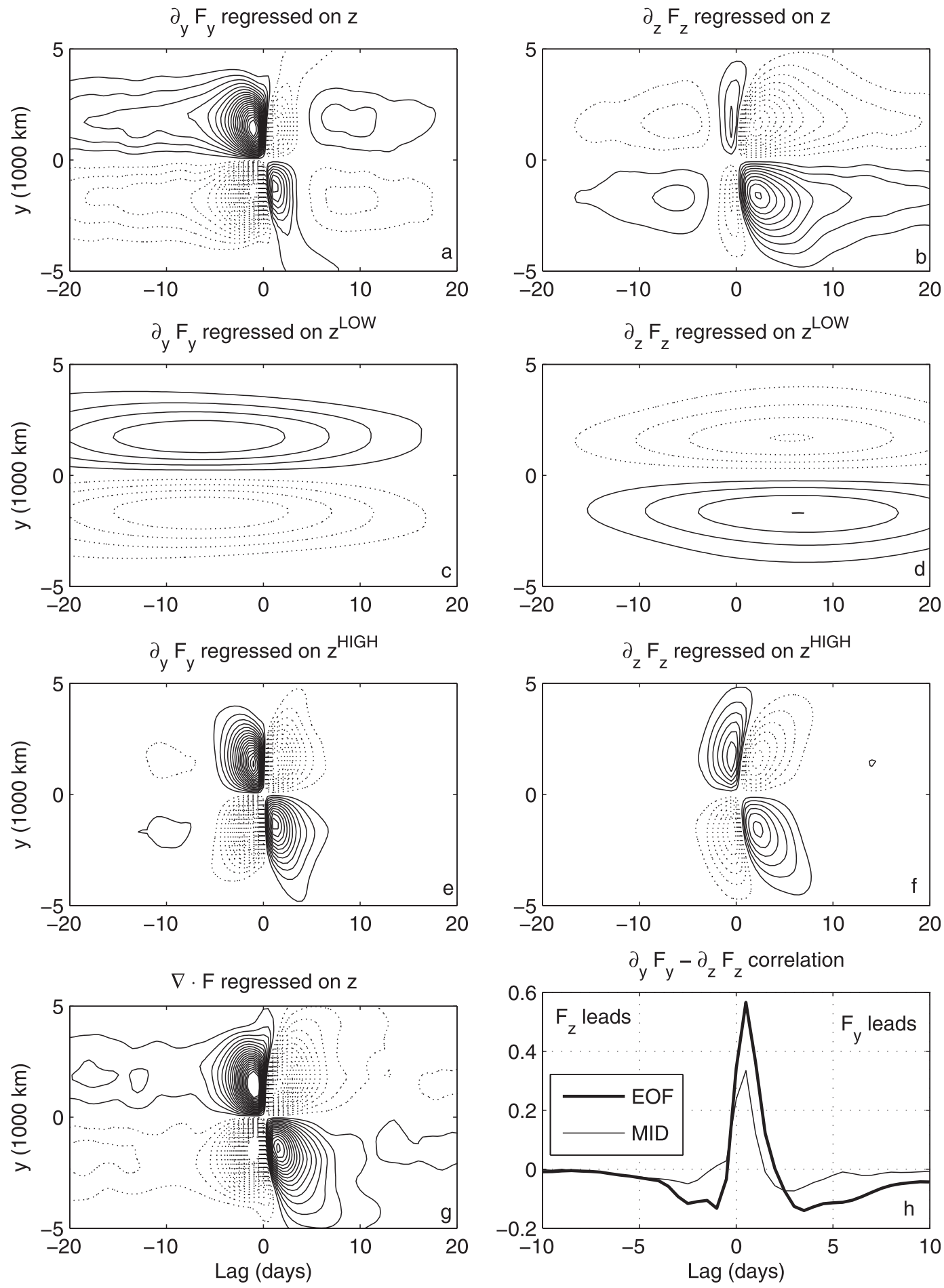

FIG. 5. Regression of (a) meridional and (b) vertical EP divergence on the normalized $z$ time series. (c),(d) As in (a),(b), respectively, but regressed on the low-frequency part of $z$. (e),(f) As in (a),(b), respectively, but regressed on the high-frequency part of $z$. (g) As in (a), but for the total EP divergence. (h) Lagged correlation between the barotropic and baroclinic components of $\boldsymbol{\nabla} \cdot F$ at midchannel (thin) and projected on $\boldsymbol{v}_{U}(y)$ (thick). The contour interval is $2.5 \times 10^{-6} \mathrm{~m} \mathrm{~s}^{-2}$ in (a)-(g) and negative contours are dotted. 

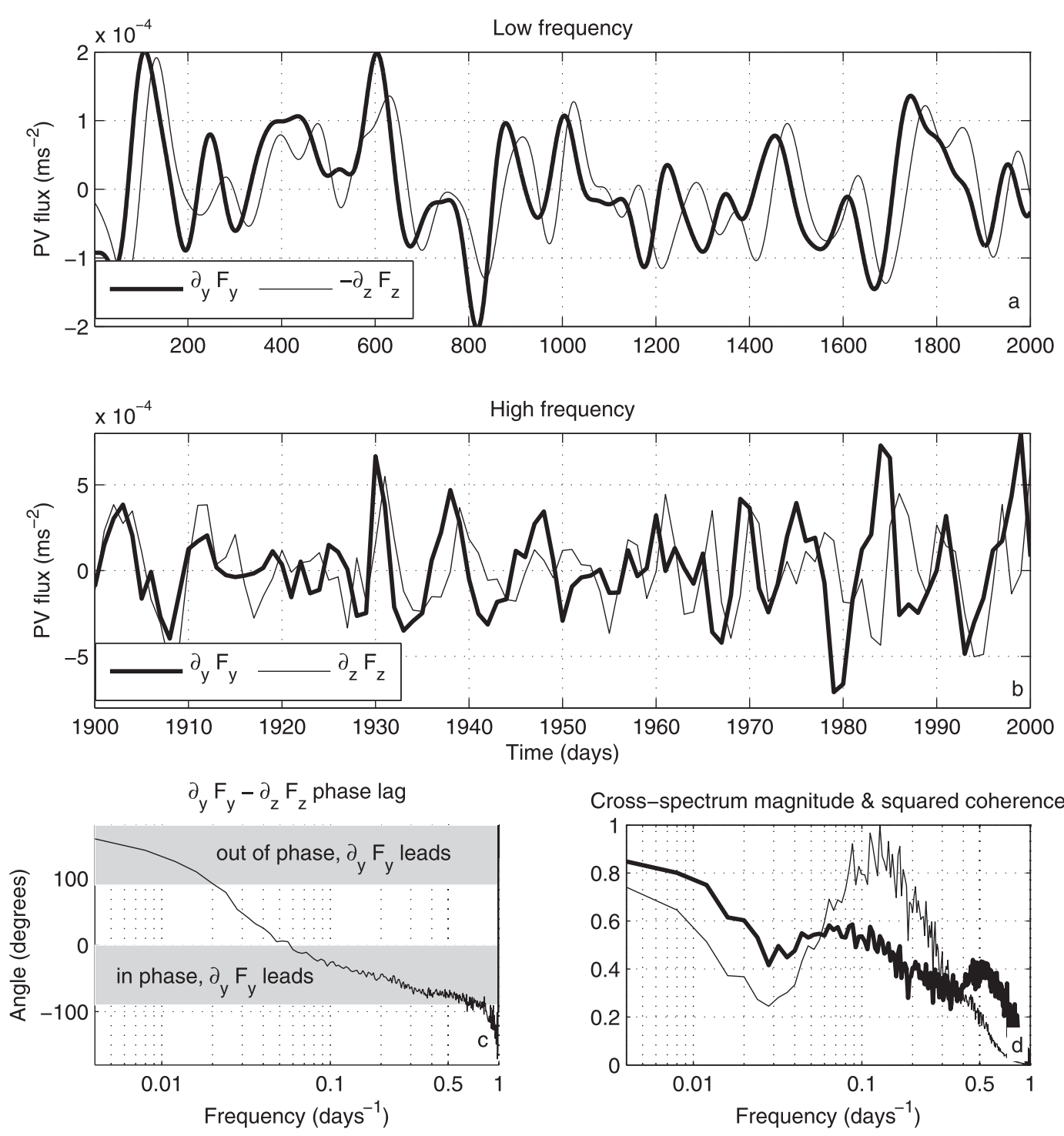

FIG. 6. (a) Sample time series of low-pass-filtered meridional divergence and vertical convergence of the EP flux. (b) As in (a), but for the high-pass-filtered meridional and vertical EP divergence. (c) Phase difference of the complex cross spectrum between $\partial_{y} F_{y}$ and $\partial_{z} F_{z}$. (d) Magnitude (thin, normalized) and squared coherence (thick) of the $\partial_{y} F_{y}$ $\partial_{z} F_{z}$ cross spectrum.

comparable (as was already apparent in Figs. 5 and 6) even though the baroclinic term clearly dominates in the climatology.

To understand the differences between the climatological/ low-frequency and time-dependent problems it is useful to consider the upper-level wave activity balance:

$$
\frac{\partial \mathcal{A}}{\partial t} \approx-\left[v_{1}^{*} q_{1}^{*}\right]-K_{\mathrm{eff}} \frac{\partial Q}{\partial Y},
$$

which is based on the hybrid Eulerian-Lagrangian formulation of Nakamura and Zhu (2010), where $\mathcal{A}$ is wave activity, $K_{\text {eff }}$ is effective diffusivity, $Q$ is potential vorticity, and $Y(Q)$ is equivalent latitude. As noted by these authors, the Eulerian eddy PV flux across latitude $Y(Q)$, $\left[v_{1}^{*} q_{1}^{*}\right]$, includes both advective and diffusive components, while the PV flux across the $Q$ contour (second term on the right-hand side) is purely diffusive. In the time mean both fluxes must be equal, implying that the climatological $\left[\overline{v_{1}^{*} q_{1}^{*}}\right]$ is constrained by effective diffusivity and by the magnitude of the PV gradient. Since effective diffusivity is usually small for geophysical flows, this sets a bound on the magnitude of the climatological $\left[\overline{v_{1}^{*} q_{1}^{*}}\right]$. In particular, the effective diffusivity over the baroclinic zone (where a mixing barrier forms; Haynes and Schuckbourgh 2000) is too small to dissipate 

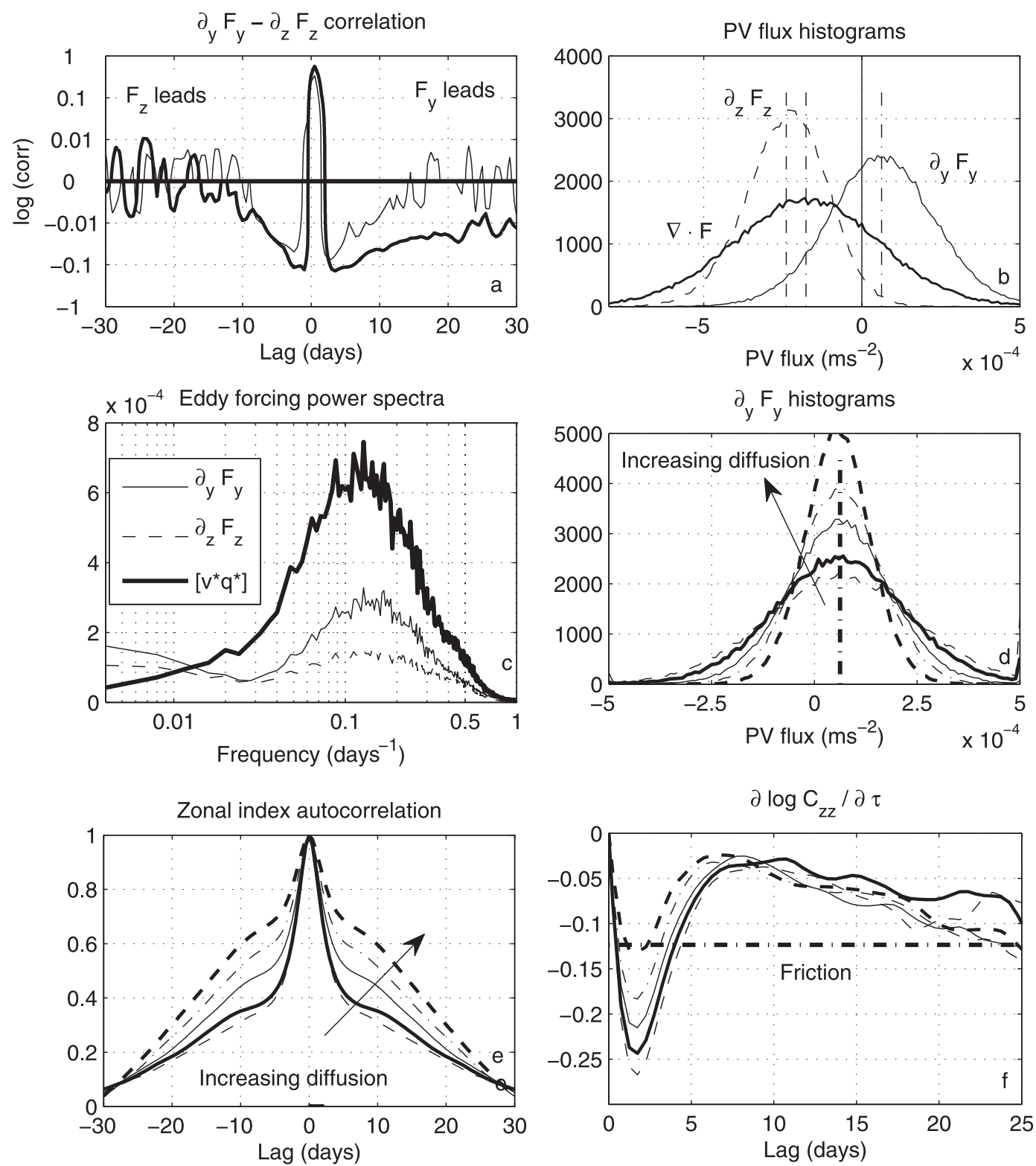

FIG. 7. (a) As in Fig. 5h, but using a logarithmic scale. (b) Histogram of the meridional (thin solid), vertical (dashed), and total (thick solid) EP divergence at midchannel. (c) Power spectra of the eddy PV flux (thick solid) and its eddy momentum (thin solid) and heat flux (dashed) components, all projected on the direction of the leading barotropic EOF. (d) Histograms of meridional EP divergence at midchannel for simulations with varying hyperdiffusion coefficients $\left[\nu=10^{15}\right.$ (thin dashed), $5 \times 10^{15}$ (control; thick solid), $2 \times 10^{16}$ (thin solid), $4 \times 10^{16}$ (dashdotted), and $8 \times 10^{16} \mathrm{~m}^{4} \mathrm{~s}^{-1}$ (thick dashed)]. (e) Zonal index autocorrelation for the same simulations as in (d). (f) Logarithmic decay rate of zonal index autocorrelation for the same simulations as in (d).

the wave activity generated baroclinically. Thus, the barotropic component of $\left[v_{1}^{*} q_{1}^{*}\right]$ will tend to compensate this baroclinic source, as waves propagate meridionally to be dissipated on the jet margins over regions with enhanced effective diffusivity.

In contrast, no such bound exists for the time-dependent problem, in which the advective component of $\left[v_{1}^{*} q_{1}^{*}\right]$ may also give rise to wave activity fluctuations. This implies that instantaneous PV fluxes can be much larger than their time-mean value, as noted above for our model. Moreover, when the instantaneous eddy PV flux is primarily balanced by transience rather than by the diffusive term in Eq. (11), the spatial structure of effective diffusivity is irrelevant and there is no preferred direction 
of meridional propagation. In this limit, the eddy PV flux is fully reversible and Eq. (11) may be approximated in spectral space as

$$
\left[v_{1}^{*} q_{1}^{*}\right](\omega)=i \omega A(\omega)-\text { Dissipation } \approx i \omega A(\omega),
$$

where $A(\omega)$ is the Fourier transform of $\mathcal{A}$, implying that the eddy forcing is fully reversible and inward (outward) import (export) of wave activity become equally likely over the baroclinic zone.

This reversible eddy forcing cannot, however, extend to the lowest frequencies (i.e., $\left[v_{1}^{*} q_{1}^{*}\right]$ cannot be white) because geometrical constraints imply that $\mathcal{A}(t)$ must be bounded when meridional eddy excursions are. If $A(\omega)$ remains finite at low frequency, Eq. (12) implies that $\left[v_{1}^{*} q_{1}^{*}\right](\omega)$ must be very small (as small as the dissipation term) when $\omega \rightarrow 0$. This is illustrated in Fig. 7c, which shows the spectrum of the eddy PV flux (projected on the leading barotropic EOF) together with the spectra of its eddy momentum and heat flux components (previously shown in Figs. 2e and $4 b$ ). The eddy PV flux spectrum has very weak amplitude at low frequency, as the redness in the eddy momentum and heat flux forcings cancel out (cf. Fig. 6a). Pseudomomentum saturation thus provides the most parsimonious explanation for the blue structure of the high-frequency eddy forcing spectra, which is associated with oscillatory eddy forcing autocorrelation as discussed in section 2b. Physically, the Eulerian eddy PV flux $\left[v_{1}^{*} q_{1}^{*}\right](t)$ must reverse as $\mathcal{A}(t)$ nears its saturation value, giving rise to the negative lobes characteristic of the autocorrelation function for all wave-mean flow interaction terms: $\partial_{y} F_{y}$ (Fig. 2b, thin line), $\partial_{z} F_{z}$ (Fig. $3 b$, thin line), and $\boldsymbol{\nabla} \cdot F$ (not shown). The structure of the $\partial_{y} F_{y}-\partial_{z} F_{z}$ cross correlation at short lags displays the same universal shape, underscoring the importance of potential vorticity dynamics.

If transience in the eddy PV flux is due to the inefficiency of diffusion to dissipate the wave activity source, as speculated above, then we would expect transience to be sensitive to hyperdiffusion. This could also have implications for zonal index persistence, as it was shown in section $2 b$ that the negative eddy forcing of the zonal index at short lags is associated with the oscillatory character of the eddy forcing autocorrelation. To investigate this we have studied the sensitivity of our model to hyperdiffusion, changing $\nu$ from $10^{15}$ to $8 \times$ $10^{16} \mathrm{~m}^{4} \mathrm{~s}^{-1}$. Although the mean state changes very little (not shown), the variability of zonal-mean flow is significantly affected. This is illustrated in Fig. 7d, which compares the observed distribution of eddy momentum convergence at midchannel for different values of the hyperdiffusion coefficient. As $v$ increases, the histograms narrow about the same mean, implying less variability. As a result of this reduced transience in the eddy forcing, the zonal index becomes more persistent at all lags (Fig. 7e). Simulations with the offline model (which neglects diffusion) produce very similar results, indicating that the autocorrelation changes with diffusion are due to changes in the eddy forcing rather than to increased damping of the zonal flow. However, note that the enhanced zonalindex persistence with increased diffusion results from reduced random variability at short lags rather than from changes in the positive eddy feedback, so that the long-lag decay rate of zonal index autocorrelation is essentially unaffected in these simulations (Fig. 7f). This example illustrates the dangers of characterizing the annular mode time scale with a single number when the eddy forcing spectrum has some structure.

\section{Disrupting the baroclinic variability}

The previous sections have shown that the internal variability of baroclinicity and zonal index are intimately related, consistent with the hypothesis of a positive baroclinic feedback for the zonal index at low frequency. In this section we investigate the sensitivity of zonal index persistence when the variability of baroclinicity is artificially inhibited.

We first consider a simulation in which temperature is restored very strongly $\left(\tau_{D}=0.01\right.$ days) to the time-mean value in the control run (rather than to radiative equilibrium). This short time scale is only used for the zonalmean heating while the diabatic damping of the eddies is kept unchanged. The mean state and eddy fluxes for this simulation are very similar to those found for the control run (not shown), consistent with the validity of diffusive closures for the mean state in this model (Zurita-Gotor 2007). However, both simulations exhibit very different variability, as zonal-mean baroclinicity must stay nearly fixed by construction in the run with strong zonal-mean restoration.

Figure 8a shows that zonal index persistence is also steeply reduced from control (cf. the thick blue and red lines), which may be explained by the deactivation of the baroclinic feedback loop in this simulation. First, the shoulder with positive $c_{m z}$ correlation when $z$ leads is no longer present for this run (Fig. 8b), consistent with the elimination of the low-frequency redness in the $m$ spectrum (Fig. 8c), the region with negative eddy forcing autocorrelation broadens (not shown) and the negative eddy forcing extends to longer lags (Fig. 8b). Additionally, the temporal relation between the eddy fluxes for this simulation is qualitatively different from that described above for the control run, and no longer consistent with the baroclinic feedback mechanism. In particular, the meridional eddy heat flux is now negatively 

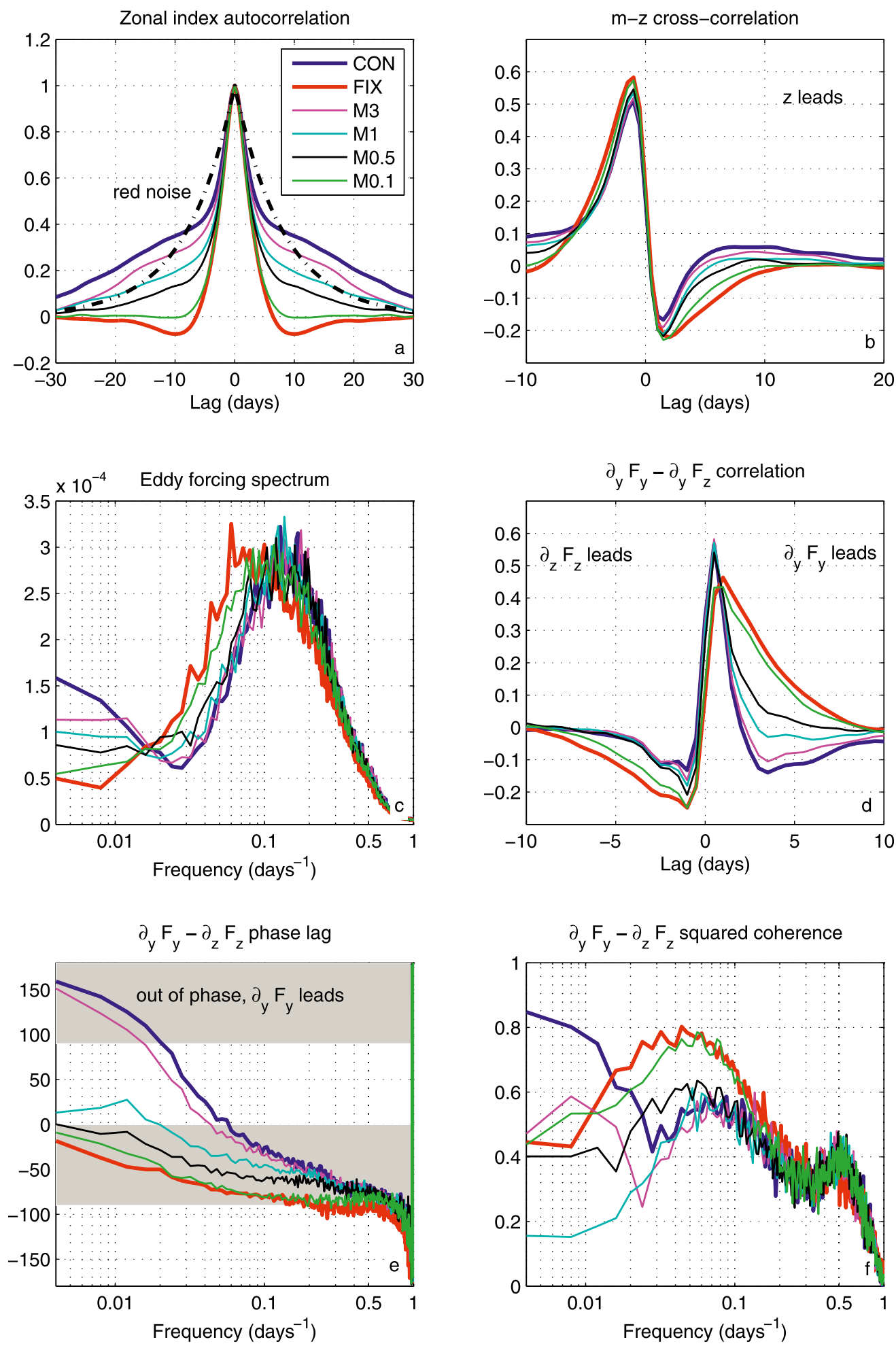

FIG. 8. (a) Zonal index autocorrelation for the control run (thick blue), the run with fixed baroclinicity (thick red), and the simulations with vertical momentum mixing with the time scales indicated in the legend. We also show the red noise limit (black dash-dotted). (b) Cross correlation between zonal index and eddy forcing for the same simulations. (c) Eddy forcing power spectrum. (d) Lagged correlation between the meridional and vertical components of $\nabla \cdot F$. (e) Phase difference of the complex cross spectrum between $\partial_{y} F_{y}$ and $\partial_{z} F_{z}$. (f) Squared coherence of the $\partial_{y} F_{y}-\partial_{z} F_{z}$ cross spectrum. 
correlated with eddy momentum convergence (i.e., $\partial_{y} F_{y}$ and $\partial_{z} F_{z}$ are positively correlated, see Fig. 8d) when the latter leads and there is no longer a spectral region with an out-of-phase relation in the $\partial_{y} F_{y}-\partial_{z} F_{z}$ cross spectrum (Fig. 8e). Finally we can see that the high spectral coherence at low frequency is also lost, implying that the bulk of the correlation between the eddy fluxes in Fig. $8 \mathrm{f}$ is now due to the synoptic eddies.

Since the fixed-baroclinicity simulation represents an extreme case, we have also analyzed the impact of disrupting baroclinicity variability on zonal index persistence in a series of experiments in which baroclinic damping is added more gradually. In the baroclinic feedback mechanism, baroclinicity is generated by the mean meridional circulation in response to the generation of westerly vertical shear by the eddy momentum fluxes and friction. We can debilitate this mechanism by means of vertical momentum mixing between the layers. Thus, we add to the right-hand side of Eq. (1):

$$
\frac{\partial q_{k}}{\partial t}=\cdots-(-1)^{k} \frac{1}{\tau_{M}} \frac{\partial M}{\partial y}
$$

where

$$
M=\left\{\left(\left[U_{1}\right]-\left[U_{2}\right]\right) \cdot \boldsymbol{v}_{U}\right\} \boldsymbol{v}_{U}(y)
$$

and $\tau_{M}$ is a mixing time scale for momentum. As indicated in Eq. (14) this momentum mixing is only applied on the zonal-mean flow, and only in the direction of the leading mode of zonal wind variability $\boldsymbol{v}_{U}$. The latter is done so as not to change the forcing of the problem, for vertical momentum mixing is equivalent to thermal damping in the quasigeostrophic framework. The meridional symmetry of the model implies that the climatology does not project on antisymmetric modes like $\boldsymbol{v}_{U}$, thus the forcing equation [Eq. (14)] has zero time mean. As a result, the time-mean state and eddy fluxes in these simulations are again very similar to those for the control run (not shown).

Diagnostics for the simulations with $\tau_{M}=3,1,0.5$, and 0.1 days have been included in all panels of Fig. 8. As the mixing time scale is reduced zonal index persistence drops, the shoulder with positive $c_{m z}$ weakens, the lowfrequency eddy forcing spectrum flattens, the spectral window with the right phase relation between $F_{y}$ and $F_{z}$ shrinks, and the results converge to those for the fixedbaroclinicity run. Although vertical momentum mixing increases the effective frictional damping on the zonal index by making the anomalous zonal wind more barotropic, this is not the main factor explaining the sensitivity of zonal index persistence on $\tau_{M}$ in Fig. 8. Simulations with the offline model keeping $\tau_{Z}=2.7 \tau_{F}$ constant produce very similar results to the full model (not shown), suggesting that the persistence drop with momentum mixing is rather due to changes in the eddy feedback.

Some recent studies of extratropical zonal wind variability using a barotropic model on the sphere (Barnes and Hartmann 2011) have found that this type of models can produce realistic zonal index variability, including a positive eddy feedback at long lags, even when the uppertroposphere wave source (the stirring) is kept fixed in latitude. This is in stark contrast with the sharp drop in zonal index persistence found in our model when baroclinicity is not allowed to move. We can understand this difference by noting that the robust covariability between barotropic and baroclinic flow in our model poses a constraint on low-frequency variability that does not exist in the barotropic model. To illustrate this, Fig. 9a compares the logarithmic decay rate of the autocorrelation functions for zonal index and baroclinicity in our control run (these were separately shown in Figs. $2 \mathrm{c}$ and 3c). As noted in section 3a, baroclinicity is less persistent than barotropic flow because it is subject to stronger negative dynamical forcing at short lags. However, it is apparent in Fig. 9a that at long lags both autocorrelation functions exhibit a similar decay rate, consistent with the high coherence between the variables at low frequency. A sensitivity analysis (results to be presented elsewhere) shows that this is a robust result in our model as parameters are varied.

The implication of this covariability is that damping the baroclinicity does not simply prevent the stirring from moving in our model: barotropic variability must also be damped when baroclinic variability is. This point is very clearly made by Fig. 9b, which shows an approximately linear relation between the long time memory of baroclinicity and zonal index in our model when vertical momentum mixing is changed. In the zonal index dynamical equation [Eq. (4)], the enhanced damping with momentum mixing can only appear through enhanced friction as modes become less top heavy and/ or through changes in $m$. The latter is the dominant effect here, underscoring how all the complexity in Eq. (4) lies on the determination of $m$. With weak momentum mixing $\left(\tau_{M} \geq 1\right)$, the low-frequency redness in $m$ is eliminated but there are very little changes in the synoptic part of the eddy forcing spectrum (Fig. 8c). Consistent with this, the positive shoulder in $c_{m z}$ weakens, while changes in the region with negative correlation are much less obvious (Fig. 8b). All this is indicative of a weakening in the long-lag positive feedback, with little changes in the negative eddy forcing at short lags. However, as $\tau_{M}$ is further reduced (and for the fixedbaroclinicity run), the synoptic eddy spectrum also changes, 

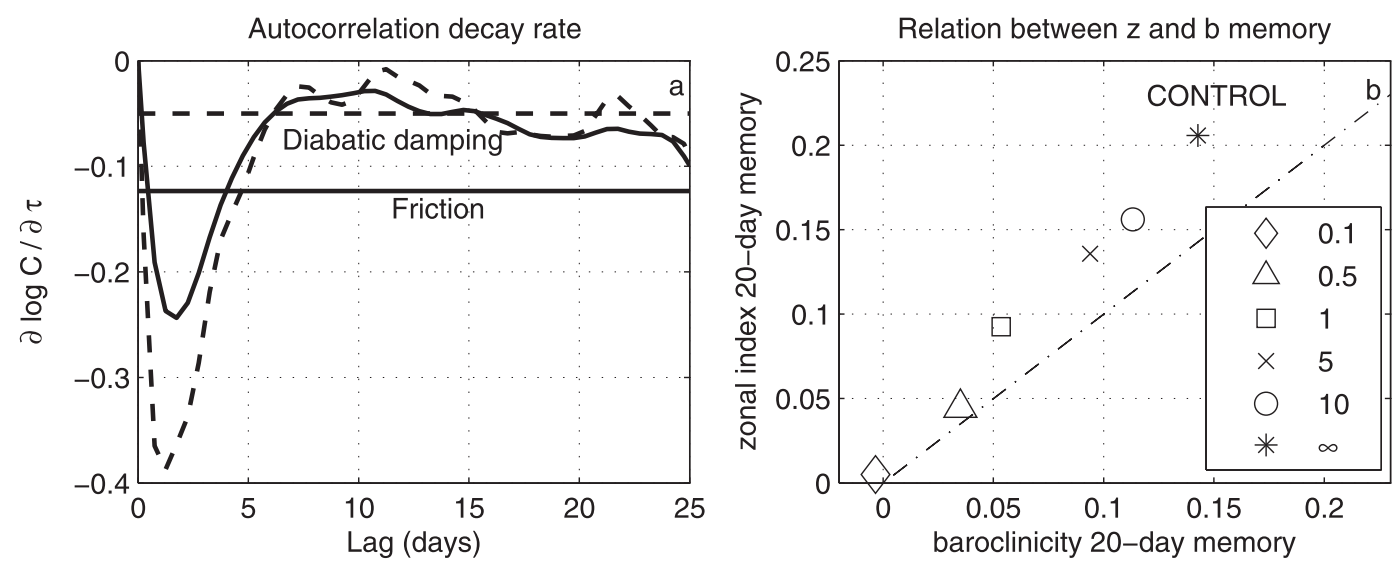

FIG. 9. (a) Logarithmic decay rate of zonal index (thick solid) and baroclinicity (thick dashed) autocorrelation compared to their respective damping time scales. (b) Zonal index memory (20-day autocorrelation) vs baroclinicity memory for the simulations with vertical momentum mixing with the time scales indicated in the legend. Note that for the two shortest time scales the shift is no longer the leading baroclinicity mode and the second mode is used instead.

broadening to lower frequencies, and the negative $c_{m z}$ extends to long lags. For these simulations, the eddy feedback actually becomes negative at long lags, as the zonal index must decay faster than its damping (frictional) time scale to vary coherently with the strongly damped baroclinicity.

\section{Summary and discussion}

In this study we have analyzed the internal variability of zonal-mean flow in a two-layer quasigeostrophic model. Despite its simplicity, this model is known to capture the basic ingredients of the extratropical circulation (Held 2005) and can also produce realistic internal variability (Lee and Feldstein 1996). In particular, we have shown that the temporal and spectral characteristics of zonal index and its eddy momentum forcing are in good agreement with observed Southern Hemisphere values (LH01). The two-layer model is the simplest model that can produce a baroclinic feedback, which makes it a very useful tool for studying the dynamics of extratropical jet variability without the complications normally arising from interactions with the subtropical jet, asymmetric meridional propagation, or vertical structure changes. Our analysis of internal variability in this model has produced the following conclusions:

1) The leading mode of baroclinicity variability is a meridional shift, which is strongly correlated with the zonal index. The correlation is maximized for short barotropic leads and the two signals are most coherent at low frequency.

2) Low-frequency baroclinicity anomalies are forced by the mean meridional circulation following the creation of vertical wind shear by the upper-level eddy momentum fluxes.

3) In contrast, the eddy heat flux by the synoptic eddies tends to damp the low-frequency baroclinicity anomalies, implying a positive correlation between baroclinicity and eddy generation at those frequencies.

4) Although the relation between the eddy heat and momentum fluxes is complicated and dominated by the fast response, both signals are very coherent at low frequency, and their phase relation is such that anomalous eddy momentum convergence precedes positive eddy heat flux anomalies.

5) The positive eddy feedback in our model weakens in the presence of vertical momentum mixing and becomes negative when the baroclinicity is not allowed to move, leading to a drastic reduction in zonal index persistence.

The governing equation for the zonal index in Eq. (4) is deceivingly simple, for all its complexity lies on the closure for $m$. It is still an open question whether one can understand the behavior of $m$ by considering upperlayer dynamics alone or baroclinic processes might also be important. The very large correlation between the zonal index and baroclinicity time series in our model $[O(0.9)$ for 100000 data points] implies a strong link between the variability of baroclinicity and barotropic flow, particularly at low frequency, when coherence is maximized-a similar relation has also been noted in observations (BZ11). The strong link between barotropic and baroclinic variability does not necessarily imply that zonal index persistence is constrained by baroclinic dynamics, as it is also possible that baroclinicity might simply respond to the zonal wind anomalies. Nevertheless, the impact of baroclinicity damping on zonal index 
persistence (section 4) provides some evidence that the problem is coupled and the interaction goes both ways, at least when baroclinicity damping is sufficiently strong. However, most of the sensitivity to momentum mixing in Fig. $9 \mathrm{~b}$ is found for time scales much faster than the diabatic time scale, which raises the question of whether baroclinic time scales might still be relevant in a more realistic setting. Although Gerber and Vallis (2007) found a decrease in zonal index persistence when the diabatic time scale is reduced in an idealized primitiveequation model, that result might be partly due to changes in the mean state rather than to the direct damping effect on baroclinic variability.

Complementary to our focus on the low-frequency positive feedback, this paper has also investigated the dynamics of the short time response. At short lags, the eddy heat flux and eddy momentum flux convergence are negatively correlated and their contributions to the upper-level PV flux add up instead of cancelling out as for the low-frequency fluxes. We argued that this lowfrequency cancellation is a consequence of the smallness of effective diffusivity, which limits the dissipation of wave activity away from critical layers. In contrast, highfrequency eddy PV fluxes need not be small and may give rise to wave activity transience. As wave-mean flow interaction terms become negatively autocorrelated at short lags, the eddy memory leads to reduced persistence of mean flow variability. The implications of the negative eddy forcing autocorrelation were already noted by LH01, who argued, however, that this feature represented an intrinsic property of the eddies and thus not a real negative feedback (i.e., the negative autocorrelation is not caused by changes in the mean flow). In our view, this feature is best understood as a consequence of the mismatch between strong wave-mean flow interaction and slow forcing/dissipation. Thus, the negative eddy forcing autocorrelation weakens with increasing hyperdiffusion.

Barnes and Hartmann (2011) have recently shown that barotropic models can produce realistic positive eddy feedbacks. In contrast, idealized baroclinic models tend to exhibit much stronger positive feedbacks than observed and unrealistic persistence (Gerber and Vallis 2007). In our model the positive feedback is also too large, but this is compensated by an excessive negative feedback because the eddies are too strong in the control setting. Since positive eddy feedbacks are weak in the barotropic model, it is plausible that the unrealistic persistence found in some idealized models might be due to the baroclinic dynamics discussed in this paper. In particular, the covariability of barotropic and baroclinic flow may lead to overly persistent zonal index variability in models with idealized thermal forcing and slow baroclinic dynamics, especially dry models (Xia et al. 2011). In this regard, note that although Newtonian relaxation only plays a minor role for the damping of lowfrequency baroclinic anomalies in our model (Fig. 4d), diabatic heating is the main damping term in observations (cf. Fig. 2c in BZ11).

The main result of our study is that low-frequency barotropic and baroclinic anomalies are very highly correlated, which may have implications for zonal index persistence. While we did not attempt to explain here why covariability is such a strong constraint in our model, the striking cancellation between the meridional and vertical components of the Eliassen-Palm convergence at low frequency (cf. Fig. 6a) provides some hints in this regard. Since the upper-level eddy PV flux is limited by the smallness of dissipation/effective diffusivity over the jet region at low frequency, persistent states with anomalous eddy generation can only be maintained when the wave activity injected at upper levels spreads to the margins of the jet to regions of enhanced dissipation. In the words of Robinson (2006), persistent, anomalous jet states must be self-maintaining.

The covariability of barotropic and baroclinic flow implies that baroclinic damping may also affect zonal index persistence, as was demonstrated here using artificial momentum mixing. The sensitivity of zonal index persistence on external parameters should provide a good measure of the role played by the different processes, barotropic or baroclinic, dynamical or nonconservative, for zonal index persistence in a realistic setting. One complication on the sphere is that the jet latitude is, in general, sensitive to external parameters, which may affect persistence (Kidston and Gerber 2010; Barnes et al. 2010). The symmetry of meridional propagation in our model may thus be useful for disambiguating changes in persistence due to changes in the internal dynamics from those due to changes in the mean state. We will analyze in a subsequent study the sensitivity of zonal index persistence on external parameters in our model, making use of the ideas and diagnostics introduced in this paper.

Acknowledgments. We are grateful to the anonymous reviewers for their helpful suggestions. This work was funded by Projects DEVIAJE (CGL2009-06948) and COMETH (CGL2012-30641) from the Ministry of Science and Innovation of Spain. EPG acknowledges support from the U.S. National Science Foundation under Project AGS1264195.

\section{REFERENCES}

Arakelian, A., and F. Codron, 2012: Southern Hemisphere jet variability in the IPSL GCM at varying resolutions. J. Atmos. Sci., 69, 3788-3799. 
Barnes, E. A. and D. Hartmann, 2010: Testing a theory for the effect of latitude on the persistence of eddy-driven jets using CMIP3 simulations. Geophys. Res. Lett., 37, L15801, doi: 10.1029/ 2010GL044144.

— variability of eddy-driven jets. J. Atmos. Sci., 68, 28932908.

,,-- D. M. W. Frierson, and J. Kidston, 2010: Effect of latitude on the persistence of eddy-driven jets. Geophys. Res. Lett., 37, L11804, doi:10.1029/2010GL043199.

Blanco-Fuentes, J., and P. Zurita-Gotor, 2011: The driving of baroclinic anomalies at different time scales. Geophys. Res. Lett., 38, L23805, doi: 10.1029/2011GL049785.

Chen, G., and P. Zurita-Gotor, 2008: The tropospheric jet response to prescribed zonal forcing in an idealized atmospheric model. J. Atmos. Sci., 65, 2254-2271.

— the persistence of the zonal index in an idealized atmospheric model. J. Atmos. Sci., 66, 3707-3720.

Edmon, H. J., B. J. Hoskins, and M. E. McIntyre, 1980: EliassenPalm cross sections for the troposphere. J. Atmos. Sci., 37, 2600-2616.

Gerber, E. P., and G. K. Vallis, 2007: Eddy-zonal flow interactions and the persistence of the zonal index. J. Atmos. Sci., 64, 32963311.

—, L. M. Polvani, and D. Ancukiewicz, 2008: Annular mode time scales in the Intergovernmental Panel on Climate Change Fourth Assessment Report models. Geophys. Res. Lett., 35, L22707, doi: 10.1029/2008GL035712.

Gómara, I., J. G. Pinto, T. Woollings, G. Masato, P. Zurita-Gotor, and B. Rodríguez-Fonseca, 2013: Rossby wave-breaking analysis of explosive cyclones in the Euro-Atlantic sector. Quart. J. Roy. Meteor. Soc., doi:10.1002/qj.2190, in press.

Hartmann, D. L., and P. Zuercher, 1998: Response of baroclinic life cycles to barotropic shear. J. Atmos. Sci., 55, 297-313.

Haynes, P. H., and E. F. Schuckbourgh, 2000: Effective diffusivity as a diagnostic of atmospheric transport. Part II: Troposphere and lower stratosphere. J. Geophys. Res., 105 (D18), 22795 22810.

Held, I. M., 2005: The gap between simulation and understanding in climate modeling. Bull. Amer. Meteor. Soc., 86, 1609-1614.

Kidston, J., and E. P. Gerber, 2010: Intermodel variability of the poleward shift of the austral jet stream in the CMIP3 integrations linked to biases in 20th century climatology. Geophys. Res. Lett., 37, L09708, doi:10.1029/2010GL042873.

Lee, S., and S. Feldstein, 1996: Mechanism of zonal index evolution in a two-layer model. J. Atmos. Sci., 53, 2232-2246.

Lorenz, D. J., and D. Hartmann, 2001: Eddy-zonal flow feedback in the Southern Hemisphere. J. Atmos. Sci., 58, 3312-3327.

Nakamura, N., and D. Zhu, 2010: Finite-amplitude wave activity and diffusive flux of potential vorticity in eddy-mean flow interaction. J. Atmos. Sci., 67, 2701-2716.
Namias, J., 1950: The index cycle and its role in the general circulation. J. Meteor., 7, 130-139.

Ring, M. J., and R. A. Plumb, 2008: The response of a simplified GCM to axisymmetric forcings: Applicability of the fluctuationdissipation theorem. J. Atmos. Sci., 65, 3830-3898.

Robinson, W., 2000: A baroclinic mechanism for the eddy feedback on the zonal index. J. Atmos. Sci., 57, 415-422.

, 2006: On the self-maintenance of midlatitude jets. J. Atmos. Sci., 63, 2109-2122.

Rossby, C. G., 1939: Relations between variation in the intensity of the zonal circulation and the displacements of the semipermanent centers of action. J. Mar. Res., 2, 38-55.

Simmons, A. J., and B. J. Hoskins, 1978: The lifecycles of some nonlinear baroclinic waves. J. Atmos. Sci., 35, 414-432.

Simpson, I. R., P. Hitchcock, T. G. Shepherd, and J. F. Scinocca, 2013a: Southern annular mode dynamics in observations and models. Part I: The influence of climatological zonal wind biases in a comprehensive GCM. J. Climate, 26, 3953-3967.

, T. G. Shepherd, P. Hitchcock, and J. F. Scinocca, 2013b: Southern annular mode dynamics in observations and models. Part II: Eddy feedbacks. J. Climate, 26, 5220-5241.

Son, S. W., S. Lee, S. B. Fledstein, and J. E. T. Hoeve, 2008a: Time scale and feedback of zonal mean flow variability. J. Atmos. Sci., 65, 935-952.

— , and Coauthors, 2008b: The impact of stratospheric ozone recovery on the Southern Hemisphere westerly jet. Science, 320, 1486-1489.

Thompson, D. W. J., and J. M. Wallace, 2000: Annular modes in the extratropical circulation. Part I: Month-to-month variability. J. Climate, 13, 1000-1016.

- , and T. Birner, 2012: On the linkages between the tropospheric isentropic slope and eddy fluxes of heat during Northern Hemisphere winter. J. Atmos. Sci., 69, 1811-1823.

Vallis, G. K., 2006: Atmospheric and Oceanic Fluid Dynamics. Cambridge University Press, 745 pp.

Xia, X., Y. Guo, and E. K. M. Chang, 2011: Diabatic damping of zonal index variations. Extended Abstracts, 18th Conf. on Atmospheric and Oceanic Fluid Dynamics, Spokane, WA, Amer. Meteor. Soc., P19. [Available online at https://ams.confex. com/ams/18Fluid/webprogram/Paper189719.html.]

Yin, J. H., 2005: A consistent poleward shift of the storm tracks in simulations of 21st century climate. Geophys. Res. Lett., 32, L18701, doi:10.1029/2005GL023684.

Zhang, Y., X.-Q. Yang, Y. Nie, and G. Chen, 2012: Annular modelike variation in a multilayer quasigeostrophic model. J. Atmos. Sci., 69, 2940-2958.

Zurita-Gotor, P., 2007: The relation between baroclinic adjustment and turbulent diffusion in the two-layer model. J. Atmos. Sci., 64, 1284-1300

_ , and R. S. Lindzen, 2007: Theories of baroclinic adjustment and eddy equilibration. The Global Circulation of the Atmosphere, T. Schneider and A. H. Sobel, Eds., Princeton University Press, 22-46. 